INPLASY

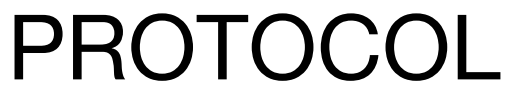

To cite: Zhou et al. Maternal anxiety and children's physical growth: A systematic review and meta-analysis. Inplasy protocol 202130056. doi: 10.37766/inplasy2021.3.0056

Received: 15 March 2021

Published: 16 March 2021

Corresponding author: Jixing Zhou

zjx314@sohu.com

Author Affiliation:

Department of Maternal, Child and Adolescent Health, School of Public Health, Anhui Medical University

Support: National Natural Science Found.

Review Stage at time of this submission: Data extraction.

Conflicts of interest:

None declared.

\section{Maternal anxiety and children's physical growth: A systematic review and meta-analysis}

Zhou, JX1'; Zhang, F2; Huang, K³ .

Review question / Objective: The prevalence of maternal anxiety during pregnancy has been increasing in recent years, which may be a significant cause of the abnormal weight of children from birth to adolescence. This study aimed to give an updated quantification between prenatal anxiety and children's weight, including birth weight, low birth weight (LBW), small for gestational age (SGA) and offspring obesity. Information sources: PubMed, EMBASE, web of science, Chinese National Knowledge Infrastructure (CNKI), Wanfang (Chinese) database and VIP (Chinese) database from 2010 to 2020.

INPLASY registration number: This protocol was registered with the International Platform of Registered Systematic Review and Meta-Analysis Protocols (INPLASY) on 16 March 2021 and was last updated on 16 March 2021 (registration number INPLASY202130056).

\section{INTRODUCTION}

Review question / Objective: The prevalence of maternal anxiety during pregnancy has been increasing in recent years, which may be a significant cause of the abnormal weight of children from birth to adolescence. This study aimed to give an updated quantification between prenatal anxiety and children's weight, including birth weight, low birth weight (LBW), small for gestational age (SGA) and offspring obesity.

Condition being studied: Normal physical development is an important foundation for individual's lifelong physiological and psychological health. Findings on the 
association between maternal anxiety and children's physical growth is conflicting. Some studies have shown a significant association between maternal anxiety and low birth weight and small for gestational age (SGA) (Gelaye et al., 2020), while other studies argued that there was no difference in birth weight between children born from mothers with or without maternal anxiety (Ibanez et al., 2012; Ravid et al., 2018). Researchers had attempted to conduct meta-analysis to illuminate the relationship between maternal anxiety and children's physical growth (Ding et al., 2014; Grigoriadis et al., 2018). However, some included studies were of cross-sectional design, mixed maternal anxiety and depression, or did not adjust for important confounders on children's physical growth. Furthermore, most of the current studies had focused on the newborn's anthropometric indications, but little emphasis has been placed on children's later-life physical growth. As some fragmented research has shown, children with anxious mothers have a higher risk of obesity (Vehmeijer et al., 2019).

\section{METHODS}

Search strategy: Two investigators (Ji-Xing Zhou, Fu Zhang) performed comprehensive literature searches independently on PubMed, EMBASE, web of science, Chinese National Knowledge Infrastructure (CNKI), Wanfang (Chinese) database and VIP (Chinese) database from 2010 to 2020. We used the following search strategy ('antenatal' or 'pregnan*' or 'prenatal' or 'gestation*') and ('anxiety' or 'mental' or 'stress' or 'emotional symptoms' or 'psychological distress') and ('child"' or 'adolescent' or 'infant' or 'toddler' or 'birth' or 'offspring' ) and ('weight' or 'obesity' or 'BMI' or 'adiposity' or 'development' or 'growth' or 'physi')'. Similar Chinese technical terms were adopted to search for eligible articles in Chinese databases.

Participant or population: A total of 28,286 studies were reviewed, and 26 studies with a combined sample size of 119,971 participants were included in the final analysis.
Intervention: No intervention.

Comparator: Pregnancy related anxiety group and non pregnancy related anxiety group.

Study designs to be included: Cohort studies.

Eligibility criteria: The following criteria were used to include published studies: (a) cohort studies; (b) maternal anxiety was assessed by means of self-reported questionnaire or structured psychiatric interview during pregnancy; (c) reporting odd ratios (ORs) with its $95 \%$ confidence intervals (Cls) after controlling important covariates or reporting mean birth weight with standard deviation (SD). Abstracts and review articles were excluded. We also excluded studies that did not distinguish depression, stress and anxiety and studies that focused on patients with gestational diabetes and other diseases during pregnancy.

Information sources: PubMed, EMBASE, web of science, Chinese National Knowledge Infrastructure (CNKI), Wanfang (Chinese) database and VIP (Chinese) database from 2010 to 2020.

Main outcome(s): The analysis included 26 studies with a combined sample size of 119,971 pairs of mother and child were included in the analysis. Maternal anxiety during pregnancy was associated with increased risk of LBW (OR $=1.23$; $95 \% \mathrm{Cl}$, 1.06-1.42), SGA (OR = 1.40; 95\% Cl, 1.201.64), and was associated with lower mean birth weight (mean difference $=-58.91 \mathrm{~g}$; $95 \% \mathrm{Cl},-89.58 \mathrm{~g}$ to $-28.25 \mathrm{~g}$ ). But the relationship between prenatal anxiety and offspring obesity was not significant (OR=1.07; 95\% Cl, 0.88-1.30). Moreover, type of gestational age and time of assessing anxiety altered the mean difference of birth weight due to prenatal anxiety.

Data management: The existence of publication bias was judged by funnel plot. The quantitative analysis of publication 
bias was performed by the Begg's test and Egger's test (Egger et al., 1997).

Strategy of data synthesis: We extracted the indicators from anxious mothers and non-anxious mothers, including the value of mean and their SD (body weight) or/and ORs and their $95 \%$ Cls (SGA, LBW, obesity). LBW was defined as birth weight less than $2500 \mathrm{~g}$. SGA was defined as newborns with birth weight below the 10th percentile of gender-specific and gestational-agespecific reference. Preterm birth was defined as infants born at less than 37 gestational weeks. Obesity was defined by International Obesity Task Force cutoffs (Cole and Lobstein, 2012), BMI z scores (Cole et al., 2000) or WHO weight standards for length/height and age in each recruited studies.

Subgroup analysis: We conducted subgroup analysis of birth weight stratified by gestational age and time of anxiety assessment during pregnancy. Regarding gestational age, two groups were set: one group included studies that only covered full-term infants or adjusted for gestational age, and the other group covered both fullterm and preterm infants. For time of anxiety assessment during pregnancy, two time windows were set as assessment before 20 gestational weeks (including the 20th week) and after 20 gestational weeks.

Sensitivity analysis: We used sensitivity analysis to investigate the stability of the outcome of meta-analysis. Every study included in this meta-analysis was deleted each time to define its influence on the pooled ORs and mean difference. If the corresponding pooled ORs and mean difference were not fundamentally altered, it would suggest that the results be statistically robust.

Country(ies) involved: China.

Keywords: anxiety; pregnancy; birth; physical growth.
Contributions of each author:

Author 1 - Jixing Zhou - The first author drafted the manuscript and extracted and analyzed the data.

Email: zjx314@sohu.com

Author 2 - Fu Zhang.

Email: 110232718@qq.com

Author 3 - Kun Huang.

Email: wuweihk8028@163.com. 